\title{
Long-term profile variability of double-peaked emission lines in AGNs
}

\author{
K. T. Lewis ${ }^{1}$, M. Eracleous ${ }^{1}$, J. P. Halpern ${ }^{2}$ and \\ T. Storchi-Bergmann ${ }^{3}$ \\ ${ }^{1}$ Department of Astronomy and Astrophysics, The Pennsylvania State University, 525 Davey \\ Laboratory, University Park, PA 16802, USA \\ ${ }^{2}$ Department of Astronomy, Columbia University, 550 West 120th Street, New York, \\ NY 10027, USA \\ ${ }^{3}$ Instituto de Fsica, UFRGS, 91501-970 Porto Alegre, RS, Brazil
}

\begin{abstract}
An increasing number of AGNs exhibit broad, double-peaked Balmer emission lines, which arise from the outer regions of the accretion disk which fuels the AGN. The line profiles vary on timescales of 5-10 years. Our group has monitored a set of 20 double-peaked emitters for the past 8 years (longer for some objects). Here we describe a project to characterize the variability patterns of the double-peaked $\mathrm{H} \alpha$ line profiles and compare with those of two simple models: a circular disk with a spiral arm and an elliptical disk.
\end{abstract}

\section{Introduction}

Approximately $20 \%$ of Broad-Line Radio Galaxies (BLRGs) exhibit broad, doublepeaked Balmer emission lines (Eracleous \& Halpern 2003). Double-peaked emission lines have also suddenly appeared in LINERs, most notably NGC 1097 (Storchi-Bergmann et al. 1993). The lines are thought to originate in the outer portions of the accretion disk $\left(\mathrm{R} \sim 100-10,000 \mathrm{r}_{g}\right.$, where $\left.\mathrm{r}_{g}=G M_{\bullet} / c^{2}\right)$. The profiles of the double-peaked emission lines are observed to vary on timescales of 5-10 yr and are a manifestation of physical changes in the outer disk. Thus, the profile variations of these lines can be used to test various models for dynamical phenomena in the outer accretion disks of AGNs.

Here, we compare the data of PKS 0921-213 with two simple, but physically motivated, models: a circular disk with a single precessing spiral arm and a precessing elliptical disk. Spiral arms are present in other astrophysical disks and provide a mechanism for removing angular momentum from the disk. An elliptical disk could result from a tidal perturbation of the disk by a massive object (e.g., a second black hole) or from the tidal disruption of a star.

Spiral Arm: The spiral arm is modeled as a circular disk with a perturbation in the emissivity pattern. The disk has the following parameters: the inner and outer radii, $\xi_{1}$ and $\xi_{2}$ (in units of $G M_{\bullet} / c^{2}$ ); the inclination angle $i$; a broadening parameter $\sigma$; and the slope of the axisymmetric emissivity law $q\left(\epsilon \propto \xi^{-q}\right)$. The spiral arm perturbation is parameterized by: an amplitude $A$; pitch angle $p$; width $\delta$; the inner radius of the arm $\xi_{s p}$; and phase angle $\phi$. Elliptical Disk: The elliptical disk model is described by many similar parameters: $i ; \sigma ; q ; \phi ; \xi_{1}$, and $\xi_{2}$, where $\xi$ is the pericenter distance. The disk is circular up to $\xi_{e}$, then the eccentricity increases linearly to a value of $e$ at $\xi_{2}$.

\section{Characterizing the Profile Variability}

Both models involve many parameters and it is difficult to determine which region of parameter space will be most successful in reproducing the observed profile variations. 

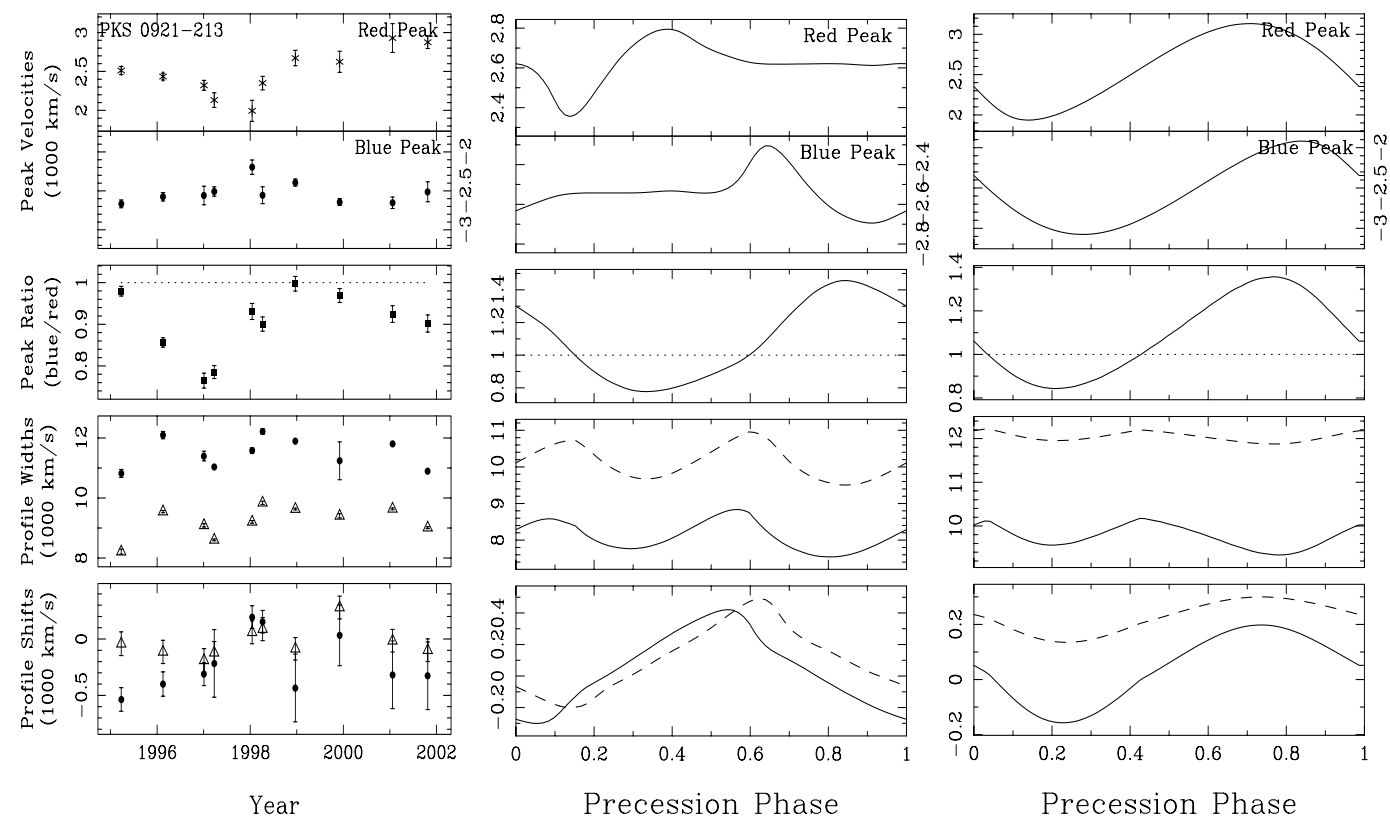

Figure 1. Variability of data and model profile properties with time. (Left) PKS 0921-213: The FWHM and FWQM, and corresponding shifts, are denoted with triangles and filled circles, respectively. (Center) Spiral Arm Model: Variation of profile properties with phase for a disk with a one-armed spiral. The model parameters are: $q=1.5 ; i=50^{\circ} ; \xi=1200-8000 \mathrm{r}_{g} ; \sigma=600$ $\mathrm{km} / \mathrm{s} ; A=5 ; \mathrm{p}=15^{\circ} ; \delta=35^{\circ} ;$ and $\xi_{s p}=1200 \mathrm{r}_{g}$. The FWHM and FWQM are denoted with solid and dashed lines, respectively. (Right) Elliptical Model: Same as the Center panel, but for an elliptical disk with $q=1.5, i=50^{\circ}, \xi=1200-8000 \mathrm{r}_{g}, \sigma=600 \mathrm{~km} / \mathrm{s}, e=0.15$, and $\xi_{e}=1200 \mathrm{r}_{g}$.

Thus, as a first step, we have begun characterizing the profile variability of our objects in a model independent way. Each profile is reduced to a set of easily measured quantities: the velocities of the red and blue peaks; the blue-to-red peak flux ratio; the full widths at half and quarter maximum (FWHM and FWQM); and the velocity shifts of the FWHM and FWQM centroids. Sets of model profiles, with a variety of input parameters, can be characterized in the same way for comparison with the data.

As an example, in Fig. 1 we show the profile parameter variations for PKS 0921213 (left), a spiral arm model (center) and an elliptical disk model (right). The circular disk parameters $\left(\xi_{1}, \xi_{2}, q, i\right.$, and $\left.\sigma\right)$ for both models are tuned to best-fit parameters of the average profile of PKS 0931-213, while the additional model parameters are simply chosen for illustration. The most striking difference between the two models is that the elliptical disk profiles always vary smoothly with phase, while those of the spiral arm model vary much more sharply. However, both models show potential for reproducing the observed profile variation. By creating "libraries" of model profiles, we will be able to quickly select a small region of parameter space to test.

\section{Acknowledgements}

KTL is supported by a NASA GRSP grant NGT5-50387.

\section{References}

Eracleous, M., \& Halpern, J. P. 2003, ApJ, 599, 886

Storchi-Bergmann, T., Baldwin, J. A., \& Wilson, A. S. 1993, ApJ, 410, L11 